\title{
S II Emission Lines in Planetary Nebulae
}

\author{
F.C. McKenna, ${ }^{1}$, F.P. Keenan, ${ }^{1}$, L.H. Aller, ${ }^{2}$, S. Hyung, ${ }^{2}$, K.L. Bell, ${ }^{3}$ and \\ C.A. Ramsbottom ${ }^{3}$ \\ ${ }^{1}$ Dept of Pure and Applied Physics, The Queen's University of Belfast; \\ ${ }^{2}$ Physics and Astronomy Department, University of California, Los Angeles; \\ ${ }^{3}$ Dept of Applied Mathematics and Theoretical Physics, The Queen's University of Belfast
}

Emission lines arising from transitions in S II have been detected in a wide variety of astronomical sources, including planetary nebulae (Hyung, Keyes \& Aller 1995). These transitions are used to derive information on emitting plasmas parameters $\left(\mathrm{T}_{e}, \mathrm{~N}_{e}\right)$ through diagnostic line ratios, although to calculate these quantities reliably, accurate atomic data must be employed, especially for electron impact excitation rates.

R-matrix calculations of electron impact excitation rates among the $3 s^{2} 3 p^{3}$ levels of S II have been derived (Keenan et al. 1996), which are found to be up to a factor of $\sim 2$ different from those of Cai \& Pradhan (1993). The present results are subsequently used to calculate emission line ratio - ratio diagrams involving the 4068, 4076, 6717 and $6730 \AA$ transitions, for a range of electron temperatures $\left(\mathrm{T}_{e}=5000-20000 \mathrm{~K}\right)$ and electron densities $\left(\mathrm{N}_{e}=10-10^{5} \mathrm{~cm}^{-3}\right)$ appropriate to gaseous nebulae. These diagrams should, in principle, allow the simultaneous determination of $\mathrm{T}_{e}$ and $\mathrm{N}_{e}$ from measurements of the [S II] lines in a spectrum. Observational data were obtained with the Hamilton Echelle spectrograph on the $3 \mathrm{~m}$ Shane Telescope at the Lick Observatory (Keyes, Aller \& Feibelman, 1990; Hyung, 1994), for a sample of planetary nebulae, including NGC 2440, NGC 7009, NGC 7027 and IC 4997. Plasma parameters deduced for this sample, including NGC 2440, NGC 7009, NGC 7027 and IC 4997, are found to show excellent internal consistency, and to be in generally good agreement with the values of $\mathrm{T}_{e}$ and $\mathrm{N}_{e}$ estimated from other line ratios in the echelle spectra, such as $\mathrm{I}(5755 \AA) / \mathrm{I}(6548+6584 \AA)$ in [N II]. These results provide observational support for the accuracy of the theoretical ratios, and hence the atomic data adopted in their derivation.

\section{REFERENCES}

Cai, W., Pradhan, A.K., 1993, ApJS, 88, 329

Hyung, S., 1994, ApJS, 90, 119

Hyung, S., Keyes, C.D., Aller, L.H., 1995, MNRAS, 272, 49

Keenan, F.P., Aller, L.H., Bell, K.L., McKenna, F.C., and Ramsbottom, C.A. 1996, MNRAS, 281, 1073

Keyes, C.D., Aller, L.H., Feibelman, W.A., 1990, PASP, 102, 59 